\title{
Design of an Attitude Stabilization Electromagnetic Module for Detumbling Uncooperative Targets
}

\author{
Albert Caubet \\ University of Strathclyde \\ 16 Richmond Street \\ G1 1XQ Glasgow, UK \\ albert.caubet@strath.ac.uk
}

\author{
James D. Biggs \\ University of Strathclyde \\ 16 Richmond Street \\ G1 1XQ Glasgow, UK \\ james.biggs@strath.ac.uk
}

\begin{abstract}
One of the challenges of Active Debris Removal is related to the tumbling of the target satellite, when the chaser spacecraft has to approach and capture it. This paper presents the ASEM (Attitude Stabilization Electromagnetic Module) concept for detumbling debris in a low Earth orbit with the intention of mitigating risk during capture operations. This paper presents a preliminary design of a system whose purpose is to stabilize a target's attitude, using an external module equipped with magnetorquers. The device, initially carried by the chaser spacecraft, is attached to the body of the target satellite. The magnetorquers are then used to detumble the target so that the chaser spacecraft can perform a safe approach prior to capture. A simplified model is used in the initial design phase to optimize the mass of the system and to size the torque rods and power source. Alternative methods for attaching the ASEM to the target from the chaser spacecraft are discussed. In addition a novel magnetic control law suitable for this application is presented. The feasibility of the concept is tested in simulations using a model based on Envisat (assuming that the ASEM is already attached to the target). Results show that a module weighing less than $20 \mathrm{~kg}$ can stabilize an 8-ton satellite initially rotating at $1 \mathrm{rpm}$ in 21 days.
\end{abstract}

\section{TABLE OF CONTENTS}

1. INTRODUCTION

2. BASELINE ARCHITECTURE .1

3. MODEL .2

4. Control laws

5. SYSTEM ELEMENTS...............................................4

6. RESULTS....................................................................

7. CONCLUSION............................................................11

REFERENCES....................................................11

\section{INTRODUCTION}

This paper proposes a novel system for detumbling passive targets in the context of Active Debris Removal (ADR). In an ADR mission, a chaser spacecraft must rendezvous with a target satellite, with the objective of modifying its orbit either for re-entry or placement into a disposal orbit. In such cases, the target satellite is uncontrolled. Several studies $[1,2]$ highlight an increasing need for ADR; for instance, every year five to ten objects should be removed from LEO in order to keep the orbit debris population constant. Moreover, the uncontrolled satellite Envisat is a major concern for ESA who may consider the possibility of deorbiting it in a controlled way. A number of techniques for ADR are being studied [3]. One of the major challenges in ADR is the tumbling or spinning of target satellites. A spacecraft approaching a tumbling satellite has a higher collision risk and the capture operation is more complex, requiring a high performance control system. Moreover, if the target is large the peak loads in a robotic arm capturing it could be relatively high. Contactless solutions have been proposed [4], which avoid the need for grasping and detumbling. However, they cannot ensure a controlled reentry and could potentially create debris in the form of small fragments. Nishida and Kawamoto [5] propose the use of a robotic arm equipped with a brush contactor to slow down the target's tumbling rate.

The purpose of this work is to outline a preliminary design of a system to stabilize the attitude dynamics of debris, reducing the risk of the capture operation. A small module with magnetorquers and a power source, initially carried by a chaser spacecraft, is attached to the target satellite. This module then acts as an external magnetic actuator that stabilizes the target's attitude by interaction with the Earth's magnetic field, damping its angular momentum. Once the target is stabilized, the spacecraft can approach it slowly to perform a safer capture. We call such a device an Attitude Stabilization Electromagnetic Module (ASEM). The use of reaction wheels for large target stabilization has been discarded as a potential detumbling actuator due to of its large mass and wheel speed requirements. However, a propulsion system could be considered as an alternative to magnetic actuators to reduce stabilization time. The de-orbit strategy itself is beyond the scope of this study, but some methodologies have been proposed in other works $[6,7]$.

A possible method to attach the ASEM to the target satellite is using a harpoon-tether system. Harpoons for ADR use have been designed and lab tested by Astrium [8]. In this scenario, a harpoon with a tether is ejected from the chaser spacecraft onto the target in close proximity flight. The chaser end of the tether is attached to the module, which then mechanically moves along the tether to reach the surface of the target. An alternative approach is for the module to use its own propulsion system, so that it can fly autonomously from the chaser to the target. A number of different control laws can be implemented in ASEM for magnetic stabilization, such as commutation (on-off) or the popular B-dot. In this paper an augmented B-dot control law, which does not require sensor information on the angular speed is proposed. 
The baseline system architecture is described in Section 2, in which the fundamental design decisions are made. Section 3 presents the model used for the sizing optimization. Additionally, in Section 4 we discuss and compare three possible control laws for attitude magnetic stabilization. In Section 5, the equations for sizing different elements of the system are described. The results of the design optimization using a simplified model, as well as full model simulations, are presented in Section 6. Section 7 provides general discussion and conclusions.

\section{BASELINE ARCHITECTURE}

The fundamental elements of the module are two torque rods and a power source. Torque rods are magnetic actuators widely used in LEO space systems and are powered by a primary battery. The concept requires that the module is physically attached to the surface of the target. As an alternative to the primary battery solution as a power source, fuel cells could be used but are heavier and more complex. Solar panels could also be used but are not as compact and a secondary battery would be required during eclipses, moreover in the tumbling scenario solar irradiation cannot be maximized. A wireless energy transfer system as a power source option-from the spacecraft to the module - could be studied in future work.

The torque rods are placed perpendicular to one another. In order to have a compact device, each rod is divided in two parts and placed in parallel, forming a square. The battery and other subsystems of the module (power distribution, onboard computer (OBC) and communications) will be placed at the centre of the module.

\section{Approach methods}

Two alternative methods are proposed to approach and attach the module to the target satellite: a harpoon-tether system and an autonomous propulsion system. The first is simple and relatively robust, however, it is a one-shot scenario and the technologies associated with harpoons fired at satellites have not been space-proven yet. The second has strong GNC requirements, but allows the chaser spacecraft to stand farther away from the target and provides the possibility of aborting the approach.

In the harpoon-tether strategy, the module can move along the tether using a simple motored pulley device. Using this method the docking procedure can then be achieved using the schematics shown in Figure 1. First, the chaser spacecraft hovers in close proximity to the target satellite. Second, it fires a harpoon towards a specified spot on the target. Third, as soon as the harpoon is firmly attached to the desired place, the module moves along the tether towards the target spacecraft. During this phase the attitude of the module could be controlled to some extent, by pivoting the point in the pulley where tension from the tether is applied. Fourth, after contact with the target satellite, the module is locked in place by applying tension to the tether, causing the legs to press against the surface.

This operation is performed with a tumbling target satellite, thus it has to be carried out quickly to avoid the tether from tangling around the satellite or twisting. Consequently, the contact speed can be relatively high and therefore a suspension system for the module is required - this aspect is discussed in Section 5.

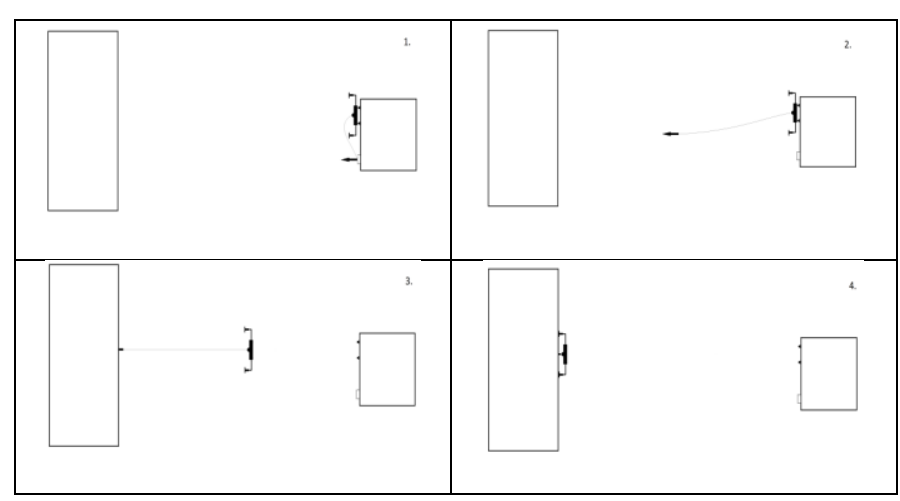

Figure 1 - Harpoon-tether approach method

An alternative method of approach for the device would be the use of a propulsion system, so that it can fly autonomously from the chaser to the target. This method would have to implement additional on-board sensors and increase the processing capacity to meet the GNC requirements. This architecture is discussed in Section 5.

The ASEM concept is represented in Figures 2 and 3. Figure 2 shows the module equipped for the pulley-tether approach method. The shock absorbers-forming the "legs" - are shown with pressure plates (the pulley is placed below the battery, thus is not shown). In Figure 3 the module features a propulsion system, including drills on the legs.

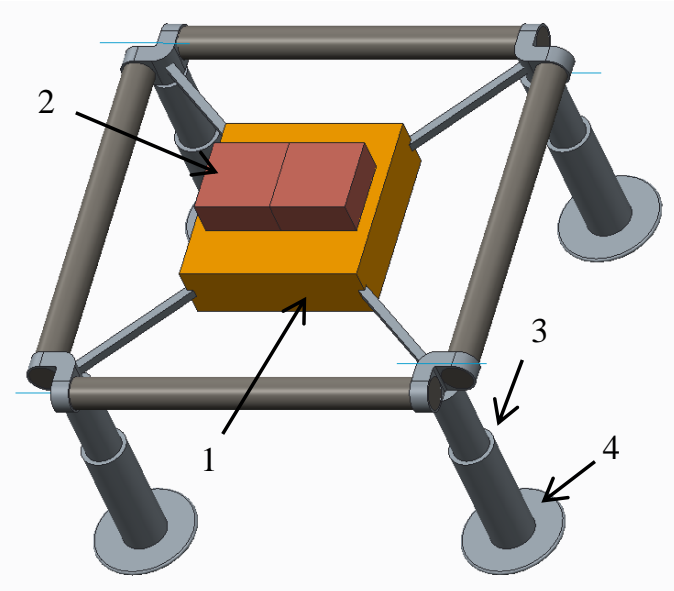

Figure 2 - ASEM with shock absorbers. 1-battery, 2subsystems, 3-suspension, 4-pressure plates (pulley not shown) 


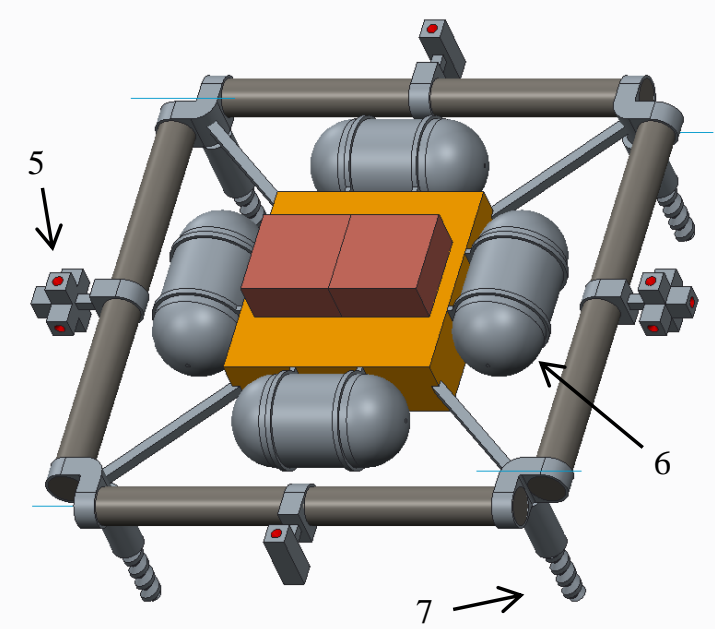

Figure 3 - ASEM with a propulsion system. 5-thruster set, 6-propellant tanks, 7-drills

\section{Attitude determination}

The controller requires information on the relative position of the target satellite with respect to the local Earth magnetic field vector. Magnetometers are simple devices that directly sense the direction of the magnetic field, but if magnetorquers are in operation, they will disturb the measurements. This implies that during the magnetometers operation the current through the torque rods should be turned off until a complete demagnetization of the core has taken place and a the B-field has been measured. Only then should the magnetorquers be turned on again. This process is inefficient and may lead to instabilities. However, we can take advantage of the fact that the chaser spacecraft is flying in close proximity to the target. One proposition is that markers on the module could be used to determine its attitude by the means of computer vision. Using magnetometers and reference sensors such as star trackers, the chaser spacecraft will obtain the direction of the B-field relative to its own attitude. Then the direction of the B-field relative to the module (thus the target satellite) will be calculated and sent to the module.

\section{MODEL}

For sizing the system, we use a simplified 2D model of a spacecraft rotating in a constant magnetic field, where a control law actuating the magnetorquers stabilizes it. With this model, analytic equations can be obtained, enabling a faster and more efficient design process. The model is inspired by Envisat which, in this scenario, is rotating at 1 rpm about the axis of largest inertia [9]. The magnetic flux density $\mathbf{B}$ (or simply magnetic field) corresponds to an orbit $1000 \mathrm{~km}$ high, considering an Earth's dipole strength of $7.96 \cdot 10^{15} \mathrm{~Wb} \cdot \mathrm{m}$. Thus, its magnitude, proportional to the dipole strength and the cube of the orbit's radius, is $B_{E}=2 \cdot 10^{-5} \mathrm{~T}$.
Figure 4 represents the model of the satellite on the $x-y$ plane, where the fixed reference frame is the body frame and the $\mathbf{B}$ field is rotating counter-clockwise. The torque rods, in black, are placed along the body axis.

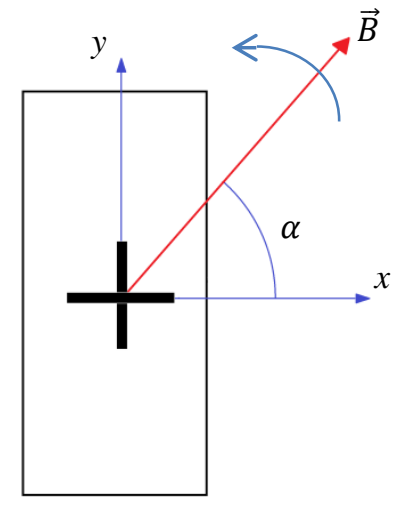

Figure 4 - Simplified model

The actuators generate a dipole moment on their axis, either in the positive or negative direction. This magnetic moment interact with the external magnetic field resulting in a torque perpendicular to both vectors:

$$
\boldsymbol{T}=\boldsymbol{m} \times B_{s}
$$

Where $\boldsymbol{T}$ is the torque vector, $\boldsymbol{m}$ is the magnetorquers' moment and $\boldsymbol{B}_{\boldsymbol{s}}$ is the Earth magnetic field relative to the body frame.

\section{Control laws}

A conventional control law for detumbling is the wellknown $B$-dot, where the magnetic moment generated by the actuators is proportional to the time derivative of $\boldsymbol{B}_{\boldsymbol{s}}$.

$$
\boldsymbol{m}=-C \dot{\boldsymbol{B}}_{\boldsymbol{s}}=\left[\begin{array}{c}
-C B_{E} \sin \alpha \dot{\alpha} \\
C B_{E} \cos \alpha \dot{\alpha} \\
0
\end{array}\right]
$$

Another option is a simple on-off control law (commutation), where the magnetorquers generate a constant magnetic moment in either direction according to the relative direction of $\boldsymbol{B}$ [10]. However, on the one hand with B-dot the control effort decreases with a decrease in angular velocity. On the other hand with commutation control there is a loss of efficiency, since full power is applied even if the actual torque is low (when $\boldsymbol{m}$ and $\boldsymbol{B}_{\boldsymbol{s}}$ are almost parallel). A compromise between the two is a modified B-dot law where the magnetic moment is only proportional to the angle with the magnetic field vector, eliminating the speed term, $\dot{\alpha}$, in Eq. (2). The result in our $2 \mathrm{D}$ model with two perpendicular magnetorquers is that the total torque magnitude is constant, thus the angular rate decreases linearly.

For a given torque rod design that provides a magnetic moment of $400 \mathrm{Am}^{2}$ and consumes $8 \mathrm{~W}$ of power, we compare the performances of the considered control laws 
when detumbling a large satellite spinning at $1 \mathrm{rpm}$. Figure 5 shows the time evolution of the angular rate during the stabilization process. In the on-off control law the mean torque is calculated as in [10]. On one hand, the B-dot law is not effective when trying to minimize the stabilization time and although the mean power used in the overall process is small, the large final time implies larger energy expenditure, as shown in Figure 6. On the other hand, the on-off or commutation law provides the minimum final time, but the fact that full power is constantly applied to the actuator, even if no effective torque is produced, makes it inefficient in terms of energy. The modified B-dot law is a fair compromise, achieving a relatively good stabilization time (Figure 5) with minimum energy expenditure (Figure 6). Therefore, the system will be sized taking this control law into account. The modified B-dot will also be used in the simulations (Section 6).

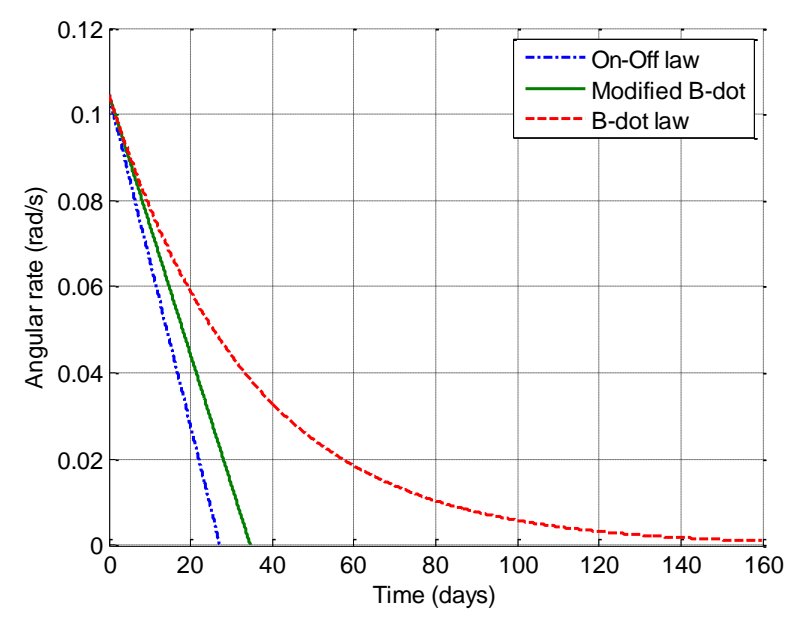

Figure 5 - Stabilization time for different control laws

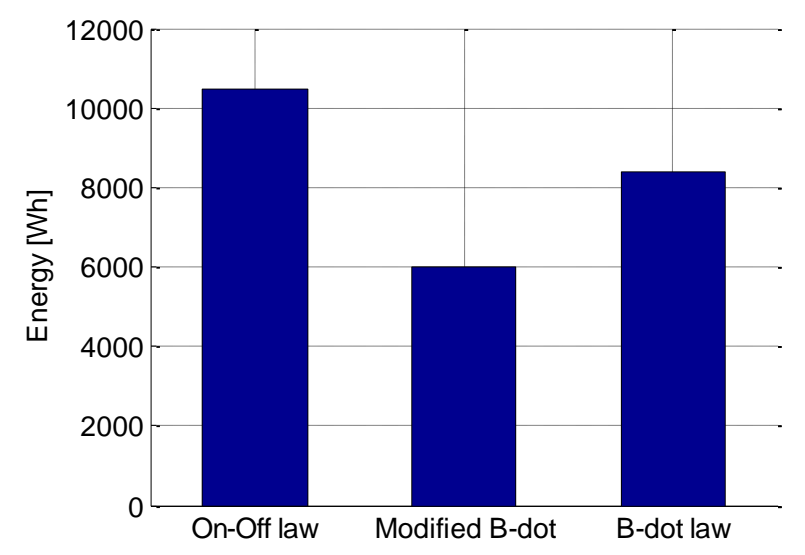

Figure 6 - Energy spent using different control laws

The control for the magnetic moment for the modified B-dot is:

$$
\boldsymbol{m}=\left[\begin{array}{c}
-C B_{E} \sin \alpha \\
C B_{E} \cos \alpha \\
0
\end{array}\right]
$$

Where $C$ is a control parameter and $\alpha$ is the angle of the magnetic field vector with respect to the $\mathrm{X}$-axis of the body frame, obtained using magnetometers. The $\boldsymbol{B}$ field relative to the body frame:

$$
\boldsymbol{B}_{\boldsymbol{s}}=\left[\begin{array}{c}
B_{E} \cos \alpha \\
B_{E} \sin \alpha \\
0
\end{array}\right]
$$

Applying Eq. (1) we have the torque (normal to the plane):

$$
\boldsymbol{T}=-C B_{E}^{2} \widehat{\boldsymbol{k}}=\ddot{\alpha} I_{3} \widehat{\boldsymbol{k}}
$$

Where $I_{3}$ is the model's inertia about the rotation axis. If $\ddot{\alpha}=\dot{\omega}_{3}$ and we integrate Eq. (5) with respect to time, the equation for the angular rate is:

$$
\omega_{3}=\omega_{3}^{i}-\frac{C B_{E}^{2}}{I_{3}} t
$$

Where $w_{3}^{i}$ is the initial angular rate. We can obtain the value of $C$ needed to stabilize the target, given a certain stabilization time $T_{s}$ and $w_{3}^{i}$.

$$
C=\frac{\omega_{3}^{i} I_{3}}{B_{E}^{2} T_{S}}
$$

In the $3 \mathrm{D}$ case, the control law is expressed as:

$$
\boldsymbol{m}=\left[\begin{array}{c}
C \sin \alpha_{1} \\
C \sin \alpha_{2} \\
0
\end{array}\right]
$$

Where $\alpha_{i}$ is the angle formed by the torque rod of the $i$ axis with the magnetic field vector.

\section{SYSTEM ELEMENTS}

In this section the expressions and assumptions for the preliminary design of several elements of the system are outlined.

\section{Torque rods}

A torque rod is a solenoid with a ferromagnetic core to amplify the magnetic moment generated when a current is applied. It is important for cores to have a high relative magnetic permeability $\left(\mu_{r}\right)$, a linear relationship between current and magnetic moment, and a high saturation point. A common core for torque rods is Hiperco $50(50 \% \mathrm{Fe}, 50 \%$ Co) with $\mu_{r}=2000$ and a density of $\rho=8385 \mathrm{~kg} / \mathrm{m}^{3}$. Although advanced materials such as Permalloy can provide a $\mu_{r}$ up to 8000 , the gain in terms of weight for the same magnetic moment is not significant.

The magnetic dipole moment $\left(\boldsymbol{m}\left[\mathrm{Am}^{2}\right]\right)$ for a simple solenoid with "air core" is simply a function of its crosssectional area $(A)$, the current $(I)$ and the number of loops of the wire $(N)$, as expressed in Eq. (8) [11]. The direction of the magnetic moment is given by the normal of the area, $\widehat{\boldsymbol{n}}$.

$$
\boldsymbol{m}=N I A \widehat{\boldsymbol{n}}
$$


However, a ferromagnetic core greatly amplifies the magnetic moment when the magnetic field generated by the solenoid orients the molecular dipoles in the material. The total magnetic field is [11]:

$$
\boldsymbol{B}=\mu_{0}(\boldsymbol{H}+\boldsymbol{M})
$$

where $\mu_{0}$ is the magnetic permeability of free air, $\boldsymbol{H}$ is the magnetic field intensity, and $\boldsymbol{M}$ is the magnetization vector field (or magnetic dipole moment per unit volume). For a solenoid with solid core, we can obtain the magnetization vector with [12]:

$$
\boldsymbol{H}=\frac{N I}{l}-N_{d} \boldsymbol{M}
$$

where $l$ is the length of the torque rod and $N_{d}$ is the demagnetizing factor, evaluated with the following expression [12]:

$$
N_{d}=\frac{4\left(\ln \left(\frac{l}{r}\right)-1\right)}{\left(\frac{l}{r}\right)^{2}-\ln \left(\frac{l}{r}\right)}
$$

Notice that this factor is a function only of the core geometry, where $r$ is the radius. Assuming a linear relation between the dipole moment and current, $\boldsymbol{B}$ and $\boldsymbol{H}$ can be related as [11]:

$$
\boldsymbol{H}=\frac{\boldsymbol{B}}{\mu_{0} \mu_{r}}
$$

where $\mu_{0}$ is the relative magnetic permeability of the core material. Combining Eqs. (10), (11) and (12) into (9) we obtain an expression showing the linearity of the magnetic field (magnitude) with the applied current (Eq. (13)).

$$
B=\frac{N I}{l} \cdot \frac{\mu_{0} \mu_{r}}{1+N_{d}\left(\mu_{r}-1\right)}
$$

The magnitude of the magnetic moment generated by the torque rod is the sum of the contributions of the solenoid windings and the magnetization of the ferromagnetic core, as expressed in Eq. (14) (let's assume $m=\|\boldsymbol{m}\|$ and $M=\|M\|)$.

$$
m=N I A+l A M
$$

Replacing the magnetization $M$ we finally have an expression of the magnetic moment as a function of the solenoid wire turns, the current, the relative permeability of the core and the geometry of the rod. For the first analysis, we will consider that the radius of the core and the solenoid itself is the same, even if the windings may have multiple layers (the contribution of the core to the magnetic moment is far larger).

$$
\begin{gathered}
m=N I A k_{1} \\
k_{1}=1+\frac{\mu_{r}-1}{1+N_{d}\left(\mu_{r}-1\right)}
\end{gathered}
$$

In order to size the torque rod, we need to know the properties of the wire for the solenoid.

$$
l_{w}=\frac{R A_{w}}{\rho_{w}}=\frac{R}{R_{w}}
$$

Eq. (17) relates the resistance with the geometry of the wire ( $l_{w}$ and $A_{w}$ are the length and cross area of the wire) and its specific resistance, either in terms of copper resistivity $\rho_{w}[\Omega \mathrm{m}]$ or resistance per metre $R_{w}[\Omega / \mathrm{m}]$ as indicated by the AWG (American Wire Gauge). For a detailed design study it would be convenient to adjust resistivity with temperature using the copper temperature coefficient $\left(\alpha=0.003862 \mathrm{~K}^{-1}\right)$.

The number of turns of the wire can be related to resistance:

$$
N=\frac{l_{w}}{2 \pi r}=\frac{R}{R_{w} 2 \pi r}
$$

If we use Ohm's law $R=V / I$, we can put the magnetic moment in terms of voltage, rod geometry and wire resistance:

$$
m=\frac{V A k_{1}}{R_{w} 2 \pi r}
$$

The magnetorquers are designed to deliver nominally the maximum magnetic moment, $m_{\max }=\left|C B_{E}\right|$. Applying more power to the solenoid to obtain a moment larger than the nominal value could lead to saturation of the core, i.e. the moment remains constant regardless of how much the intensity is increased.

The design values described in this section are for one torque rod; to size the complete system we must take into account the two magnetorquers.

\section{Battery}

Primary batteries are not rechargeable, but have a much larger specific energy ( $\mathrm{SE}$, in $\mathrm{W} \cdot \mathrm{h} / \mathrm{kg}$ ) than rechargeable secondary batteries. The battery should be sized to store enough energy to detumble the target satellite using magnetorquers. With the mean power required by the torque rods and the stabilization time, the mass of the battery of a certain type can be approximated simply using its SE value. Similarly, the energy density can be described as the energy per unit volume $(\mathrm{ED}$, in $\mathrm{W} \cdot \mathrm{h} / \mathrm{l})$, thus the approximate volume of the battery can also be obtained.

It is desired to select a battery with high SE and ED, as well as a good temperature range. Lithium-carbon monofluoride (CFx) primary batteries are very efficient, low weight and compact. According to a NASA manual [13], they have a specific energy up to $625 \mathrm{Wh} / \mathrm{kg}$ and an energy density up to $1070 \mathrm{Wh} / 1$ (specifications of more modern models of this type of battery, e.g. the ones manufactured by Quallion, are similar).

The battery mass and volume are calculated using the mean value of the total power. In our model, the power applied to 
each torque rod is a sinusoidal wave, phased 90 degrees according to the control law (Eq (3)). Since the magnetic moment is proportional to the power, and if $P$ is the maximum power applied to a single magnetorquer, then the total power as a function of the angle $\alpha$ with the magnetic field vector $\boldsymbol{B}_{\boldsymbol{s}}$ is:

$$
P_{\text {total }}=P(-\sin \alpha)+P(\cos \alpha)
$$

The mean value, denoted by $\langle\cdot\rangle$, and considering the absolute value of the total power, is calculated as follows:

$$
\left\langle P_{\text {total }}\right\rangle=P \frac{1}{\pi} \int_{\frac{\pi}{4}}^{\frac{5 \pi}{4}}(-\sin \alpha+\cos \alpha) \mathrm{d} \alpha=P \frac{2 \sqrt{2}}{\pi}
$$

\section{Shock absorber}

In the harpoon-tether scenario, the module makes contact with the surface of the target at a significant speed. Shock absorbers, namely springs, are used to bring the module to rest on the target. Their mass and properties are estimated in this section.

In order to avoid a collision between the chaser spacecraft and the tumbling target, a safety distance shall separate them. In our particular case study, Envisat's largest dimension (including solar panels) is $26 \mathrm{~m}$ long. Thus, if we set a distance of $30 \mathrm{~m}$ from the centre of mass of Envisat, the chaser spacecraft will always be a minimum of $17 \mathrm{~m}$ away from any part of the satellite.

As the satellite is rotating and there is a tether attached to it, it is desirable to get the module from the chaser as quickly as possible to avoid twisting the tether and tangling up around the satellite. Since we assume an angular rate for the target of $1 \mathrm{rpm}$, in our design we require that the module shall arrive to the surface of the target in 15 seconds (i.e. a quarter of a rotation).

Those requirements set the final speed with which the module will get to the target. Assuming constant acceleration, i.e. the motor pulls from the tether applying a constant tension, the final speed is calculated in Eq. (22).

$$
v_{f}=\frac{2 x_{f}}{t_{f}}
$$

If the distance to the satellite surface is $x_{f}=30 \mathrm{~m}$, the arrival speed will be $v_{f}=4 \mathrm{~m} / \mathrm{s}$. Then with the mass of the module it is straightforward to calculate the kinetic energy at contact time, energy to be absorbed by four springs placed at the tips of the structure. The springs will be designed so that we obtain an estimate of their mass. The basic equations for spring design [14] are shown in Appendix A.

Titanium is the chosen material, since it makes high performance springs due to its elastic properties, resistance and weight. The kinematic energy of the module will be transformed into elastic potential energy by the springs. At the moment of the impact the honeycomb structure may bend, absorbing part of the kinetic energy. This effect is not assessed in the design. Matching kinetic and elastic energies we have:

$$
M_{t} v_{f}^{2}=N_{s} F_{\max } \Delta x
$$

Where $M_{t}$ is the total mass of the module and $N_{s}=4$ is the number of springs. The equations above can be combined so that five design parameters are needed to determine the spring's mass: final speed $v_{f}$, total mass $M_{t}$, wire diameter $d$, outer spring diameter $D_{\text {outer }}$, and number of coils $n_{a}$. Two constraints on the geometry are set: first, the maximum slenderness ratio $\frac{L_{\text {free }}}{D}$ is limited to 4 (to prevent bucking during compression efforts), and second, the spring index $C=\frac{D}{d}$ is limited between 3 and 15 to avoid buckling and excessive internal stress. Preliminary results show that the spring mass is minimized if the diameter and the number of coils are minimized. Thus, we set those to relatively small but reasonable values: $D_{\text {outer }}=4 \mathrm{~cm}$ and $n_{a}=5$. Finally, an optimizer finds the wire diameter that minimizes mass.

A relevant solution is the relationship between the mass of the module and the mass of all dumpers, for a given speed of the module at contact (Figure 7). The sum of spring masses has a value of nearly 180 grams for a $20-\mathrm{kg}$ module. An also relevant figure of merit is the maximum force each spring exerts onto the surface, ranging from $500 \mathrm{~N}$ for a 15 $\mathrm{kg}$ module to $1100 \mathrm{~N}$ for a 30-kg module (assuming also a contact speed of $4 \mathrm{~m} / \mathrm{s}$ ). This shock force could bend or pierce the structure; should this event be avoided, pressure plates can be added at the base of the dumpers. Also, the dumpers shall be designed so that they stay compressed at their maximum displacement or they are restored in a controlled way, in order to prevent the module to bounce back as a consequence of an elastic shock. Within the module mass specified in those figures, the spring length is $14.2-14.5 \mathrm{~cm}$.

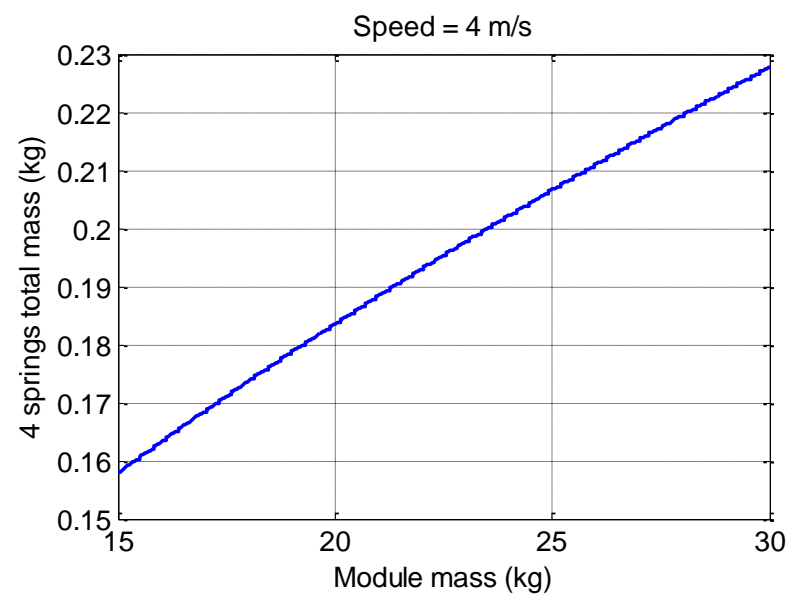

Figure 7 - Springs mass

Notice that the springs have been sized according to rather strong constraints. If the safety distance constraint was relaxed from $30 \mathrm{~m}$ to $25 \mathrm{~m}$, the final module speed would be 
$3.3 \mathrm{~m} / \mathrm{s}$, and this halves the maximum force made by the spring. Regarding the mass of the springs, results show that it is only a small fraction of the total mass of the module and their optimization is not critical, allowing for relatively large safety factors.

\section{Propulsion system}

An alternative option to the harpoon-tether system is using a propulsion system on the ASEM. This option would allow the module to fly on its own from the chaser onto the target satellite. In this scenario, the module incorporates a full propulsion system, i.e. a set of thrusters, propellant tanks and pipes. Also, it shall feature a robust and performing GNC system, in order to maneuver safely to the specified spot on the surface of the tumbling target. The chaser spacecraft can help in this task, using image processing to determine the attitude and position of both the module and the target (in this case the spacecraft shall have direct vision of the module during the maneuver). Finally, once it makes contact with the surface, drills on the legs lock the module in place, in a similar way as Rosetta's comet lander [15].

The advantage over the harpoon-tether architecture is robustness, in the sense that, first, several attempts to approach the target are possible provided there is enough propellant, and second, the chaser spacecraft can stand farther away from the tumbling target. The downside is an increase in mass and complexity.

To size the propulsion system, delta-v estimations are done. Since the satellite is tumbling, the approach maneuver should be performed relatively fast, to minimize the risk of being hit by any part of the satellite body. In this preliminary work, we require that the module, hovering 20 $\mathrm{m}$ away from the target, shall approach it in 5 seconds and arrive with zero velocity, assuming a linear path. This corresponds to a delta-v of $16 \mathrm{~m} / \mathrm{s}$. Applying a factor of two, to allow for maneuver contingencies or a second approach attempt, we round the delta-v requirement to $30 \mathrm{~m} / \mathrm{s}$. Notice that this specification is on the high end of the sizing range. In a detailed mission design, the maneuver can be optimised using simulations with an effective path planning algorithm, thus reducing the required mass of the system.

Finally, using basic kinematics, for a module of $20 \mathrm{~kg}$ the required thrust is $64 \mathrm{~N}$. Since the maneuver involves a tumbling target, it is desired to have 6 DOF control, in the translational and attitude motions. A set of 14 thrusters distributed on four corners of the module structure can provide full control (see Figure 3). Provided that four thrusters are aligned with the main motion direction, each thruster shall provide a minimum of $16 \mathrm{~N}$.

The two considered options for the propulsion system are cold gas and monopropellant hydrazine [16]. The first is very simple and reliable, but has low $\mathrm{I}_{\mathrm{sp}}$. Thrust is provided by high pressure gas, which requires relatively bulky tanks but low weight and simple thrusters. The second option uses an exothermic decomposition of the propellant (usually hydrazine, $\mathrm{N}_{2} \mathrm{H}_{2}$ ), which provides a higher $\mathrm{I}_{\mathrm{sp}}$. It is still a relatively simple and reliable system, but less than cold gas. However, the high toxicity of hydrazine is a major issue.

Considering the thrust requirements specified above, the mass of the monopropellant thrusters is about $200 \mathrm{~g}$, while the mass of a typical small cold gas thruster is about $25 \mathrm{~g}$. Thus, just the added mass of the set of monopropellant thrusters $(2.8 \mathrm{~kg})$ is higher than a cold gas system with the same specifications - including tanks. Therefore, cold gas is selected, with nitrogen as the propellant due to its storage density, performance and lack of contamination concerns.

In order to estimate the required propellant mass, Tsiolkovsky rocket equation is used:

$$
\Delta v=I_{s p} g_{0} \ln \left(\frac{m_{d r y}+m_{p}}{m_{d r y}}\right)
$$

Where $m_{p}$ and $m_{d r y}$ are the propellant and dry mass. If we choose a delta-v of $30 \mathrm{~m} / \mathrm{s}$ with a $20-\mathrm{kg}$ module (dry mass), the mass of the propellant is $0.92 \mathrm{~kg}$. For the same specifications, the volume of the propellant (nitrogen) is 3.3 litres. The mass of the spherical tanks is calculated using Eq. (25). It relates the tensile stress of the material $(\sigma)$, the pressure $(P)$, radius $(r)$ and thickness $(t)$.

$$
\sigma=\frac{P r}{2 t}
$$

Typical operating pressure is 135 atm. Given the estimated volume, if we divide it in four tanks, each one will have a radius of $5.8 \mathrm{~cm}$. Selecting aluminium as the tank material we have the value for its allowable stress, and thickness can be evaluated. Finally, with the material density the mass is determined: each tank has a mass of $0.1 \mathrm{~kg}$. If we add a $20 \%$ of the overall tankage mass for mounting hardware and propellant management devices [16], the total tank and pipe system weighs $0.48 \mathrm{~kg}$. As a final remark, if ammonia was used as propellant instead of nitrogen it would reduce overall tankage mass in $0.2 \mathrm{~kg}$, since it is stored in liquid form. However, this type of propellant does not allow for high mass flow rates [17].

Summing up the mass of propellant, tanks and thrusters, the overall cold gas propulsion system mass is estimated in 1.75 $\mathrm{kg}$, which represents an $8.75 \%$ of the $20-\mathrm{kg}$ module mass used for this sizing example.

\section{RESUltS}

\section{Design optimization}

Using the equations described above, we can obtain analytically the mass of the system formed by the torque rods (wire and core) and the battery. Our aim is to minimize the mass while accomplishing the requirement of detumbling a specific target, rotating at a certain angular rate, within a reasonable amount of time. 
First, the wire, core and battery properties are selected. The ferromagnetic core of the torque rod is Hiperco 50, described in Section 5.1. Each magnetorquer is treated as a single rod in the calculations, although in the final module configuration they are cut and placed in pairs. The battery is a CFx type, described in Section 5.2. Suitable wires for torque rods are 24,28 or 32 AWG. 24 AWG is selected as it minimizes mass for a solenoid of the same properties.

Then there are four design variables left to size the system: the desired stabilization time $\left(T_{s}\right)$, the power of the system $(P)$, and the geometry of the rod (length $l$ and radius $r$ ).

Using the various equations described previously in this paper, the design process is:

1. Problem parameters:

a. Orbit altitude, $h=1000 \mathrm{~km}$

b. Initial angular rate, $\omega_{3}^{i}=6 \mathrm{deg} / \mathrm{s}=1 \mathrm{rpm}$

c. Target inertia, $J_{3}=227630 \mathrm{~kg} \cdot \mathrm{m}^{2}$

2. Design parameters:

a. Frozen:

i. Wire $24 \mathrm{AWG}, R_{w}=0.084 \Omega / \mathrm{m}$, $\rho_{w}=8940 \mathrm{~kg} / \mathrm{m}^{3}$

ii. Battery CFx, $S E=625 \mathrm{Wh} / \mathrm{kg}, E D=$ $1070 \mathrm{Wh} / \mathrm{l}$

iii. Core Hiperco 50, $\mu_{r}=2000, \rho_{c}=$

b. Variables: $8385 \mathrm{~kg} / \mathrm{m}^{3}$

i. Stabilization time, $T_{S}$

ii. Power, $P$

iii. Torque rod geometry, $l$ and $r$

3. Design flow:

a. Magnetic moment $m_{\max }$ to stabilize the target in a certain time $T_{S}$

b. Required voltage given a certain torque rod design

c. Number of wire turns

d. Torque rod mass (wire mass and core mass) e. Battery mass (function of $T_{s},\left\langle P_{\text {total }}\right\rangle$ and $S E$ )

f. Total mass (battery and 2 torque rods)

Additional parameters can be obtained to help in the design, such as the number of layers of the wire windings, the maximum value of the intensity, or the number of battery cells required, to name a few.

Having frozen the wire, core and battery types, there are four parameters left to be determined, that minimize the mass of the system. Since the design is done with analytical equations, we can use an exhaustive search to ensure global optimality and gain insight of the design process. Results are obtained using a combination of the four design variables. Since the analytical equations of the simplified model are used, millions of designs are obtained within minutes. The main analysis of the data is based on obtaining the power and rod geometry that minimize the system mass, for every stabilization time $T_{s}$.

The angular rate for this case study has been set to $1 \mathrm{rpm}$, or $6 \mathrm{deg} / \mathrm{s}$. While there is no exhaustive study on the tumbling rates of inoperative satellite in LEO, Nishida and Kawamoto [7] consider that $1 \mathrm{rpm}$ falls into the "medium" range of angular rate, requiring some slow-down before capture. Castronuovo [6] highlights that a robotic arm could capture a Soyuz (7 tons) spinning at $4 \mathrm{deg} / \mathrm{s}$, with a peak torque of $80 \mathrm{Nm}$.

\section{Preliminary results}

The conclusion on rod geometry after the first results is that the mass is minimized with the maximum slenderness ratio $\lambda=l / d$ (where $d$ is the rod diameter). Nevertheless, the slenderness of a torque rod has to be limited to avoid strong flexible modes and buckling. A maximum slenderness ratio of 34 is selected. This value is in the higher range of existing torque rods, e.g. Microcosm models. The length of the rod shall not be very high since it is desirable to design a compact device. We limit it to $l=850 \mathrm{~mm}$, thus the diameter of the core is $d=25 \mathrm{~mm}$. 


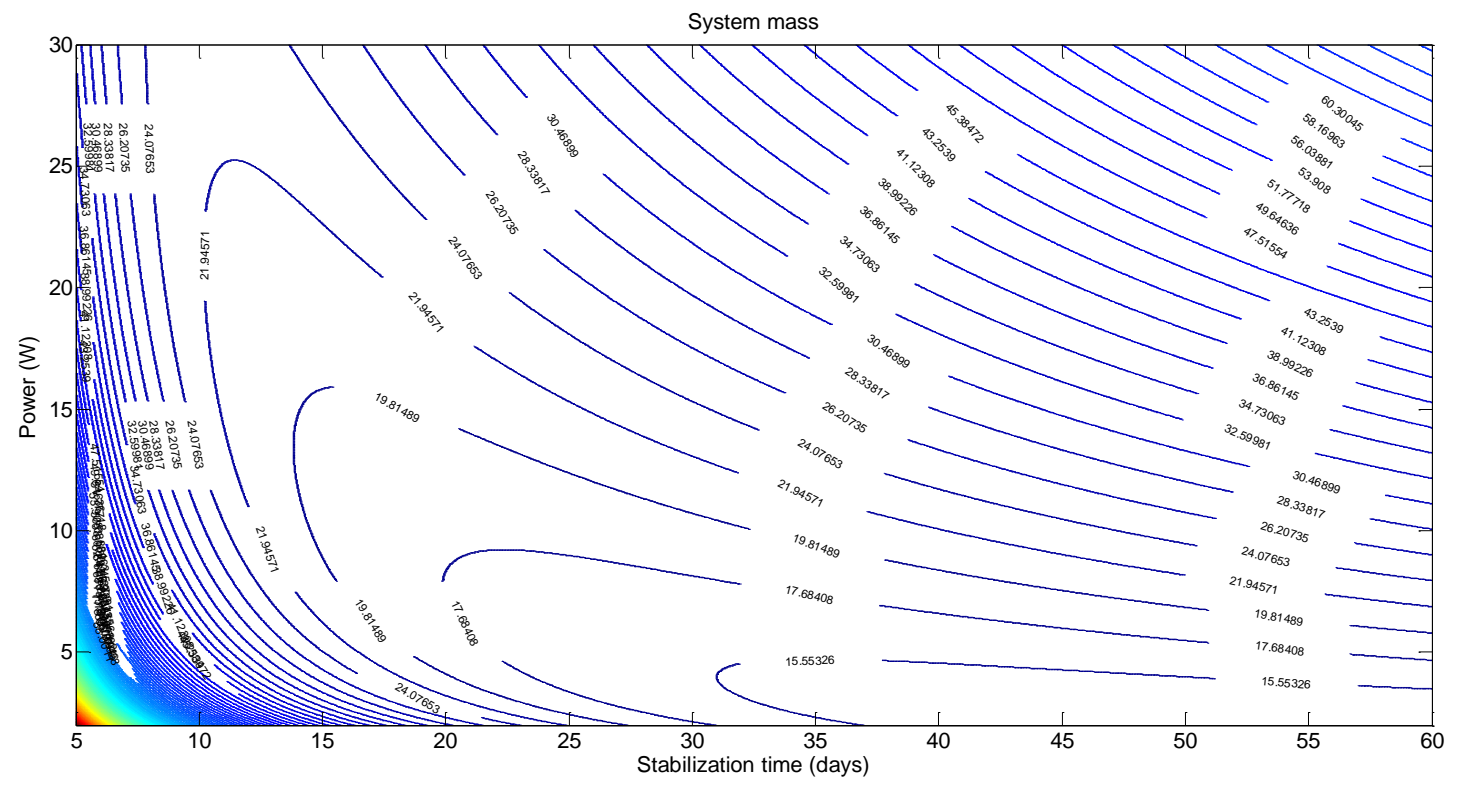

Figure 8 - Mass required for different values of delivered power and stabilization times

Figure 8 shows the total mass of the system for values of power and stabilization time, given the selected rod geometry.

It is clear that for a given stabilization or detumbling time there is a certain power that minimizes mass, and the minimum-mass power decreases logarithmically as the time increases. From another point of view, for a given arbitrary power, there is a minimum system mass corresponding to a determined stabilization time. Figure 9 shows the mass of the different elements of the system with respect to stabilization time for a constant, arbitrary power. (The "Rods mass" is the sum of both magnetorquers' mass, and stabilization and settling terms are used interchangeably.) The mass of the battery increases linearly with time as the mass of the torque rods decreases logarithmically.

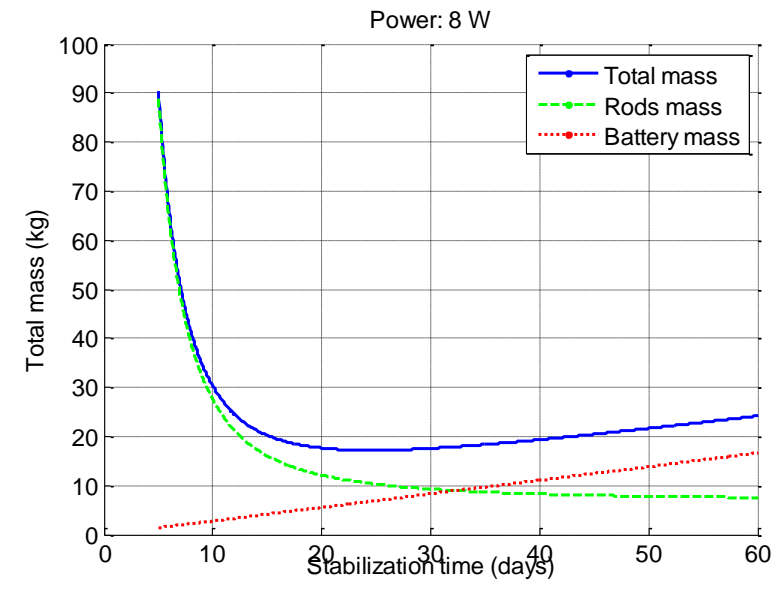

Figure 9 - Breakdown mass for a given power

Additionally, given a nominal magnetic moment and rod geometry, the nominal power sets the necessary number of turns of the wire.

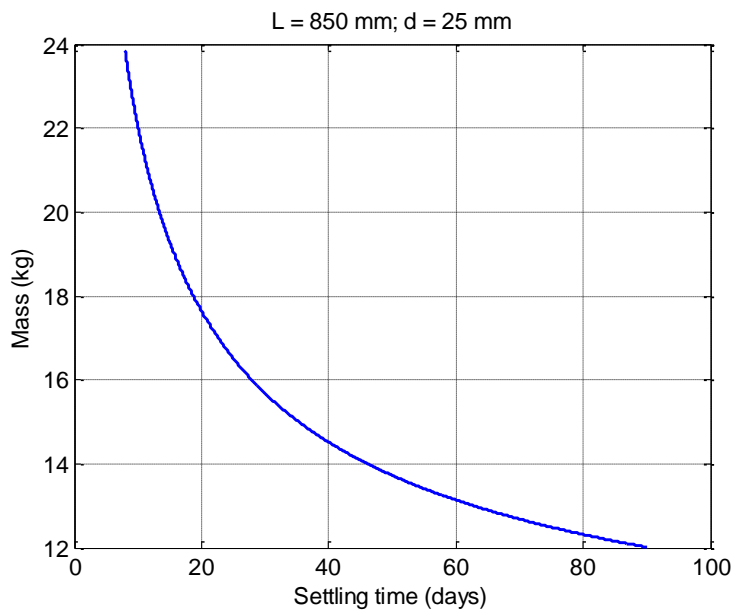

Figure 10 - Minimized total mass

Figure 10 represents the absolute minimum mass of the system for every stabilization time (this is, for every time the optimal power is selected). The mass decreases as the stabilization time increases, making a trade-off decision. At higher stabilization times, the mass decrease rate is low. Eventually it rises again, but the time for the optimal mass is too high for practical purposes. The breakdown of the different elements of the system, including the battery volume is shown in Figure 11 (single rod mass is plotted). In the figures' timespan (8-90 days), the number of winding layers in the torque rods ranges from 15 to 5 . The required magnetic moment is shown in Figure 12. 


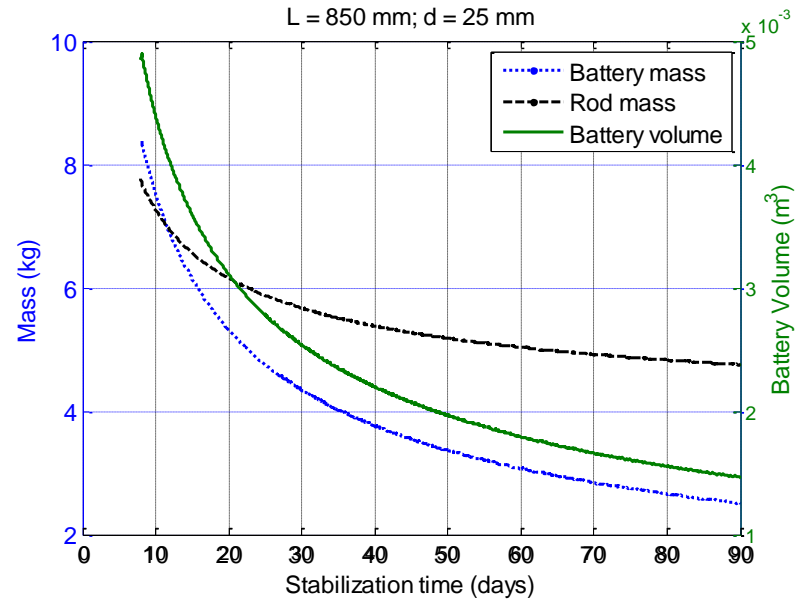

Figure 11 - Minimized mass breakdown and battery volume

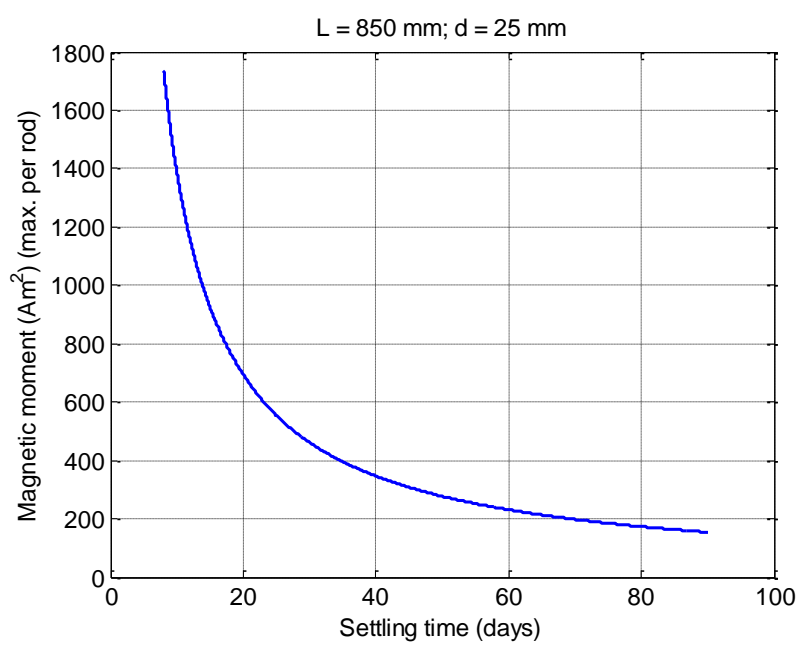

Figure 12-Maximum magnetic moment required

Selecting the stabilization time is now the main design decision. It is desirable to minimize the time, while keeping the mass of the system low. On one hand, mass minimization is relevant not only for launch costs, but also for the fact that a lighter module will be more agile when reaching the target satellite from the chaser spacecraft. On the other hand, high stabilization times increase the operational cost, the amount of time that the spacecraft flies in close proximity to the target (thus spending more fuel), and the risk of failure associated to long operation times.

\section{Simulation results}

In order to optimize the design, we have previously worked with a 2D model and simplified dynamics. In this section, simulations of a full 3D model are done, including the orbit dynamics and a model of the Earth magnetic field [10]. The atmospheric drag and solar radiation pressure are not considered, since their effects are not relevant in the simulated scenario.
An orbit similar to that of Envisat is considered, with an inclination of 99 degrees and an altitude of $1000 \mathrm{~km}$. The moments of inertia in the principal axis of the target satellite are $\boldsymbol{J}=[9361,225960,227630]^{T} \mathrm{~kg} \cdot \mathrm{m}^{2}$. We assume that the module is placed on the largest side of the satellite, and the two magnetorquers are aligned with its first and second axis. The initial angular velocity of the satellite is $\boldsymbol{\omega}_{0}=$ $[0.07,0.04,0.07]^{T} \mathrm{rad} / \mathrm{s}$, whose norm is approximately 1 $\mathrm{rpm}$. The magnetorquers in the simulation provide a maximum magnetic moment of $400 \mathrm{Am}^{2}$. As shown in Figure 13, the actuators progressively stabilize the tumbling of the target. When a threshold of $0.01 \mathrm{rpm}$ is achieved, the magnetorquers are turned off and a small, residual angular velocity remains.

Since the Earth magnetic field relative to the satellite varies with the orbit's position, two magnetorquers are sufficient for stabilization purposes. In the simulation, the satellite is stabilized in 21.5 days; by the $10^{\text {th }}$ day the satellite tumbling is already stabilized in two axes while the remaining angular rate about the third axis is linearly decreasing. According to the 2D model, the stabilization time using two $400 \mathrm{Am}^{2}$ magnetorquers is about 35 days (Figure 12). In this simulation the final time is lower, however results show that the energy spent is higher, thus a $4.6 \mathrm{~kg}$ primary battery is required as opposed to the $4 \mathrm{~kg}$ one used in the simplified model (considering an $S E=625 \mathrm{Wh} / \mathrm{kg}$ ). This proves that the simplified 2D model is useful to obtain an initial guess of the design parameters, but they have to be refined using a realistic model of every special case. Generally speaking, the battery shall be oversized by a certain safety factor to allow for uncertainties and for longer stabilization times than expected.

In the previous simulation, the combined mass of the battery and magnetorquers is $15.6 \mathrm{~kg}$. We add an estimate of $15 \%$ of this mass for structure and the rest of minor subsystems. If the propulsion option is selected, the overall mass of the module is $19.7 \mathrm{~kg}$; in the harpoon and tether scenario, the added mass of the shock absorbers $(0.2 \mathrm{~kg})$ and pulley system (estimated $0.5 \mathrm{~kg}$ ), the total mass is $18.6 \mathrm{~kg}$.

If another magnetorquer is incorporated (orthogonal to the other two), simulations with the same initial conditions show that there is no significant improvement in the final stabilization time, while the total energy spent is higherrequiring $6.5 \mathrm{~kg}$ of battery, $2 \mathrm{~kg}$ more than the twomagnetorquer option.

At low inclination orbits, the external magnetic field vector does not change as much with respect to the orbit position. In those cases the magnetic stabilization is not as effective - in the ideal case of an equatorial orbit on the geomagnetic reference frame, one axis would remain uncontrolled. For instance, in an orbit with an inclination of 30 degrees, the same magnetorquers take 45 days to stabilize the satellite, using a battery of $9.7 \mathrm{~kg}-5 \mathrm{~kg}$ more 


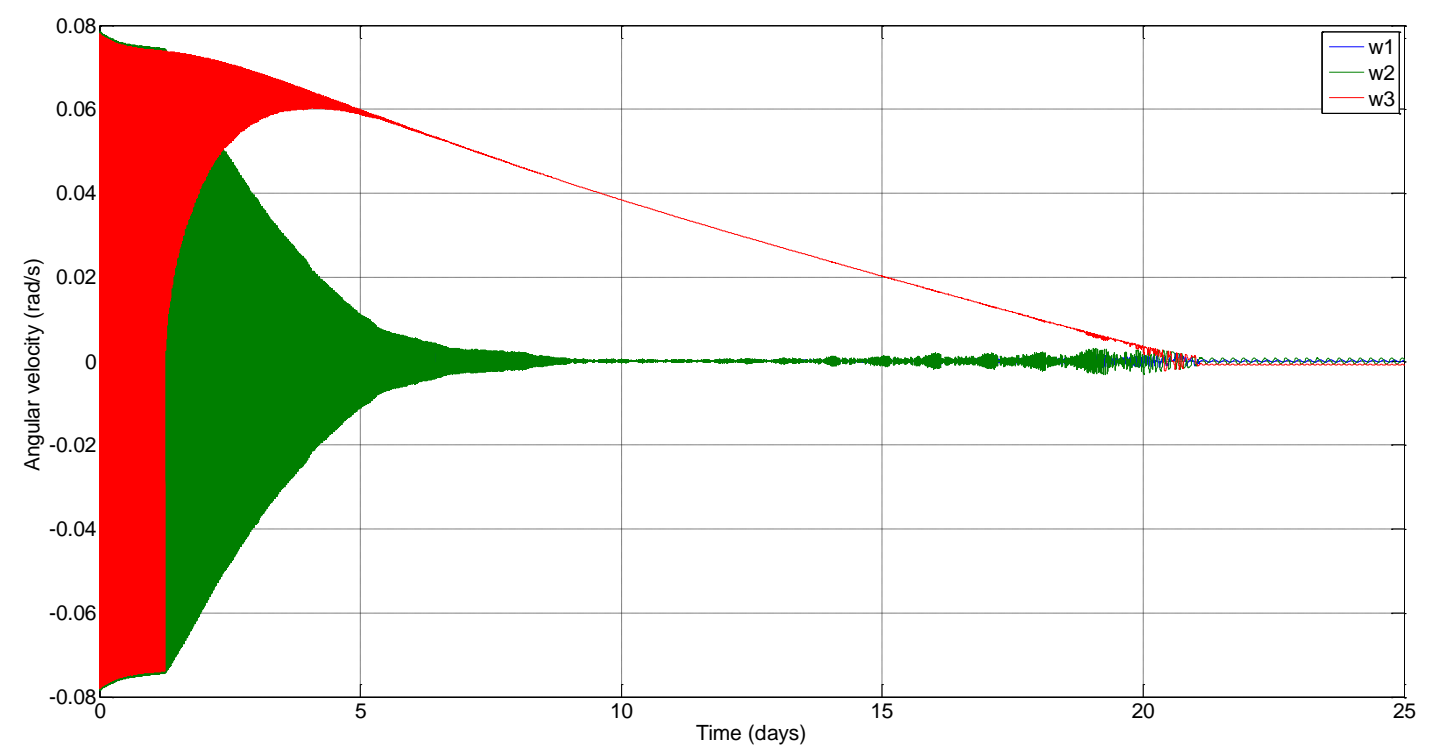

Figure 13 - Evolution of the angular velocity of an 8-ton target at an orbit $1000 \mathrm{~km}$ high and inclined 99 deg., using two $400 \mathrm{Am}^{2}$ magnetorquers

than in the high inclination orbit scenario. If a third magnetorquer is incorporated in the low inclination case, the improvement in stabilization time is only of 3.5 days, at the expense of 4.1 more $\mathrm{kg}$ of battery mass and the extra weight of the torque rod.

\section{CONCLuSion}

A simplified model has been used to optimize the sizing of the ASEM system. Two different strategies to attach the module to the target have been analysed and sized using approximate estimations. Since their weight difference is not significant when compared to the total system, mass is not a driver when selecting one or the other. Simulations have been undertaken with a model based on the Envisat satellite. Additionally, a modified B-dot control law has been proposed, and a method for attitude determination using the chaser spacecraft's sensors has been discussed.

The simplified model is able to provide an order of magnitude of the sizing parameters; however, simulations on every special case are required for a detailed system design. Simulation results show that the time required for stabilization varies strongly with the inclination of the orbit. Nevertheless, the majority of the satellites in LEO are not placed in low inclination orbits, the most problematic for the use of this system. The case study in this paper suggests that the ASEM concept is able to stabilize a large satellite such as Envisat in a reasonable amount of time, using a module weighting less than $20 \mathrm{~kg}$. A total angular velocity of $1 \mathrm{rpm}$ has been assumed; very high angular velocities of the target could require an important increase in the system's mass.
The module is small and light enough to be carried on the chaser spacecraft. Due to the relatively small size and weight potentially more than one module could be carried on the chaser either for redundancy or to use on several targets. An ASEM system may allow the design of the chaser spacecraft to reduce the constraints on agility and size-since it doesn't have to maneuver to approach and capture a tumbling target. Moreover, when the angular momentum of the target is high (e.g. in large, tumbling satellites), the ASEM would save the extra mass associated with a robust robotic arm requiring to withstand high torque loads. Overall, the concept presented intends to provide a complementary system to existing ADR systems for characterizing ADR mission architectures.

\section{REFERENCES}

[1] J.-C. Liou, N.L. Johnson, Hill, N.M. Controlling the growth of future LEO debris populations with active debris removal. Acta Astronautica 66, 648-653, 2010

[2] J.-C. Liou. An Active Debris Removal Parametric Study for LEO Environment Remediation. Advances in Space Research 47, 1865-1876, 2011 
[3] B. Bastida, H. Krag. Strategies for Active Removal in LEO, Proceedings of the 5th European Conference on Space Debris, ESA SP-672, 2009

[4] E. Ahedo, M. Merino, C. Bombardelli, H. Urrutxua, J. Pelaez, and L. Summerer. Space debris removal with an ion beam shepherd satellite: target-plasma interaction. $47^{\text {th }}$ IAA/ASME/SAE/ASEE Joint Propulsion Conference \& Exhibit. AIAA, July 2011

[5] S. Nishida, S. Kawamoto. Dynamical Simulations for Space Debris Capture, SICE Annual Conference, 22832286, 2011

[6] M. Castronuovo. Active Space Debris Removal-A Preliminary Mission Analysis and Design, Acta Astronautica 69, 848-859, 2011

[7] S. Nishida, S. Kawamoto. Strategy for Capturing of a Tumbling Space Debris, Acta Astronautica 68, 113-120, 2011

[8] J. Reed, J. Busquets, and C. White. Grappling system for capturing heavy space debris. 2nd European Workshop on Active Debris Removal, 2012

[9] M. Degener. Experiences in Large Satellite Modal Survey Testing. Proceedings European Conference on Spacecraft Structures, Materials and Mechanical Testing, 659-664, 1999

[10] J. R. Wertz. Spacecraft Attitude Determination and Control. Kluwer Academic Publishers, 1990, ISBN 90277-1204-2

[11] D. A. de Wolf. Essentials of Electromagnetism. Cambridge University Press, 2001, ISBN 0-321-66281-8

[12] W.L. Black. Design of Electromagnetic Torque Rods. Technical Note 1965-45, Lincoln Laboratory MIT, 1965

[13] B. J. Bragg, J. E. Casey, J. B. Trout. Primary Battery Design and Safety Guidelines Handbook. NASA Reference Publication 1353, 1994

[14] F. Haideri. Design of Machine Elements. Nirali Prakashan, 2005, ISBN 9-380-06432-2

[15] J. Biele, S. Ulamec. Capabilities of Philae, the Rosetta Lander. Space Sci Rev 138: 275-289, 2008

[16] J. R. Wertz, W. J. Larson. Space Mission Analysis and Design. Microcosm Press/Kulwer Academic Publishers, $3^{\text {rd }}$ ed., 1999, ISBN 1-881883-10-8

[17] A. Anis. Cold gas propulsion system-An ideal choice for remote sensing small satellites. Remote sensingAdvanced techniques and platforms, InTech, Chapters, 2012, ISBN 978-953-51-0652-4

\section{BIOGRAPHY}

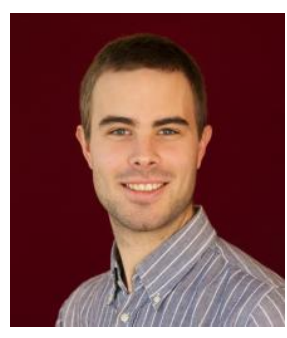

Albert Caubet received a M.Sc. in Aeronautical Engineering from Polytechnic University of Catalonia (UPC-BarcelonaTech) in 2011. He has worked at the French Space Agency (CNES) in trajectory optimization for the Rosetta mission lander, and in a semi-analytic orbit propagator for space debris assessment (STELA). In 2013 he was awarded a Marie Curie grant and started a PhD in spacecraft autonomy and control at the University of Strathclyde in Glasgow, $U K$.

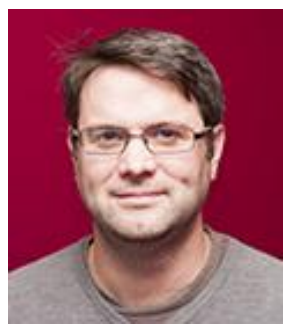

James Biggs received the B.Sc. degree in Mathematics from the University of Sussex, UK in 1998, the M.Sc. degree in Nonlinear Dynamics and Chaos from University College London, London, UK in 1999 and the Ph.D. degree from the University of Reading, UK. He has industrial experience as a quantitative analyst in both the defence and financial sectors. He is currently an associate director of the Advanced Space Concepts Laboratory and senior lecturer at the University of Strathclyde, Glasgow. Presently his research interests are focused on using dynamical systems theory, control theory and differential geometry to develop optimal guidance and control algorithms for autonomous systems such as spacecraft, unmanned air vehicles and underwater vehicles. 
APPENDIX A

\begin{tabular}{|l|c|}
\hline Elastic constant: & $k=\frac{G d^{4}}{8 D^{3} n_{a}}$ \\
\hline Mean spring diameter: & $D=D_{\text {outer }}-d$ \\
\hline Maximum displacement: & $\Delta x_{\max }=L_{\text {free }}-\left(n_{a}-2\right) d$ \\
\hline Maximum force: & $F_{\max }=k \Delta x_{\text {max }}$ \\
\hline Rise angle: & $\theta=\tan ^{-1}\left(\frac{L_{\text {free }}}{n_{a} \pi D}\right)$ \\
\hline Wire length: & $L_{\text {wire }}=\pi D\left(\frac{n_{a}}{\cos (\theta)}+2\right)$ \\
\hline Spring mass: & $M_{\text {spr }}=\rho L_{\text {wire }} \frac{\pi}{4} d^{2}$ \\
\hline
\end{tabular}

Where $G$ and $\rho$ are the material's shear modulus and density, $d$ is the wire diameter, and $n_{a}$ is the number of active coils. 